\title{
Material attributes that define performance and efficiency of spaceborne mirrors
}

Tony Hull, Janina Krieg, Ralf Jedamzik, Thomas Westerhoff

Tony Hull, Janina Krieg, Ralf Jedamzik, Thomas Westerhoff, "Material attributes that define performance and efficiency of spaceborne mirrors," Proc. SPIE 11852, International Conference on Space Optics — ICSO 2020, 118524Q (11 June 2021); doi: 10.1117/12.2599806

SPIE Event: International Conference on Space Optics - ICSO 2021, 2021, Online Only 


\section{International Conference on Space Optics-ICSO 2020}

Virtual Conference

30 March-2 April 2021

Edited by Bruno Cugny, Zoran Sodnik, and Nikos Karafolas
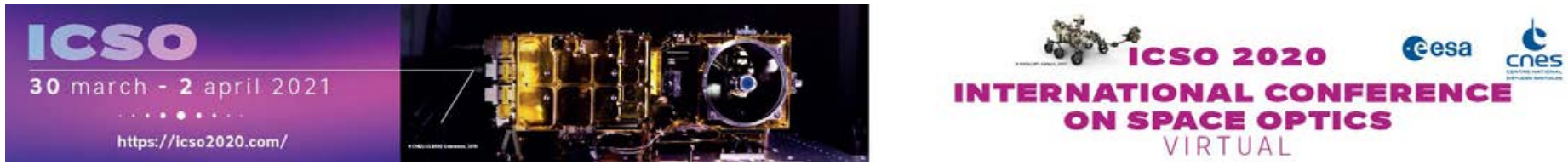

\section{Material attributes that define performance and efficiency of spaceborne mirrors}

\section{Cesa isoporacedings ecnes}




\title{
Material attributes that define performance and efficiency of spaceborne mirrors \\ HULL Tony ${ }^{1}$, KRIEG Janina ${ }^{2}$, JEDAMZIK Ralf ${ }^{2}$, WESTERHOFF Thomas ${ }^{2}$ \\ ${ }^{1}$ Department of Physics \& Astronomy, University of New Mexico US \\ ${ }^{2}$ SCHOTT AG, Mainz, DE
}

\begin{abstract}
In addition to the classical parameters used for mirror substrate material selection, we discuss several other attributes critical to implementation of spaceborne mirrors for various environments. Often trades are either limited to the material and approach the designer has used in the past, whether or not it is optimum for the application, or are based on primary factors like specific stiffness and transient response. We look further at mission-critical attributes, including fracture mechanics, temporal drifts, deterministic implementation to design, inhomogeneity, anisotropy, polishability, compatibility with advanced coatings, and space heritage. We extend these considerations into discussion of increasing emphasis on designing to cost, available sizes and possible delivery timescales.
\end{abstract}

\section{INTRODUCTION}

Optimal design of spaceborne telescope assemblies begins with a clear science flow down, environment (orbit) selection and especially matching telescope requirements and environments with the optimal material. In a companion paper at this conference, we address other factors critical to the implementation of significantly lightweighted mirrors with advanced aspheric forms. The optimal material choice depends on available supporting data, going beyond the first order mechanical and thermal characteristics. Thus, cost, schedule, Technology Readiness Level (TRL), risk assessment and expected error budget margins are all linked with a detailed knowledge of first and second order material attributes. For example, in the thermal domain, far more is needed than coefficient of thermal expansion (CTE) and thermal diffusivity of the material, but also the homogeneity and isotropy of CTE. Decisions should include both the environment the mission will experience, and the innate dimensional stability of the materials to be used. Of these telescope decisions, none has a bigger effect on system performance, complexity, risk and cost than the selection of mirror material. The relative importance of various material parameters is evolving as well. For example, with the advent of Heavy Lift Launch Vehicles, extreme lightweighting driving mirror architecture of the last several decades may be relatively less important than other factors. With interest in constellations of moderate-sized telescopes, Design-To-Cost becomes an enabling factor much as performance factors. Past and present weighting of relative importance of both available materials and available processes may now be different. Contemporary mirror design should regard as critical specific aspects:

a) the determinacy of making the substrate,

b) effort required in subsequent optical processes

c) thermal compatibility with available metering materials over environmental range, and

d) the complexity, cost and risk of the required thermal management system.

We reference material alternates to the space heritage material ZERODUR ${ }^{\circledR}$, since ZERODUR ${ }^{\circledR}$ has been extensively characterized with results published by SCHOTT [2-8, 10-13].

\section{ENVIRONMENTS AND MATERIAL RESPONSE}

\section{Space environment factors:}

Earth borne telescope environments are distinct from those in space. Some of the factors that a designer of spaceborne telescopes must consider and accommodate are expressed in Table 1. 
Table 1. The Space Environment Effects influencing the mirror material selection: two elements are rated as strongly relevant

\begin{tabular}{|c|c|c|c|}
\hline \# & Environment Element & Relevance & Notes as applies to mirror material selection \\
\hline 1 & Vacuum & Minor & Thermal influence difference without convective heat transfer \\
\hline 2 & Launch and gravity release & Major & Structural design, modulus and testing \\
\hline 3 & Mass to space & Moderate & Presently of lower priority with reduction of cost $/ \mathrm{kg}$ \\
\hline 4 & Plasma environment & Minor & Electrical charging minor within a telescope assembly \\
\hline 5 & Space radiation environment & Moderate & $\begin{array}{l}\text { High levels cause compaction, with the possibility of changing the } \\
\text { high precision optical surface }\end{array}$ \\
\hline 6 & $\begin{array}{l}\text { Equilibrium temperature, } \\
\text { gradients and transients }\end{array}$ & $\begin{array}{l}\text { Most } \\
\text { Significant }\end{array}$ & $\begin{array}{l}\text { In our experience, thermal changes constitute a major cause of } \\
\text { dimensional instability and resulting degradation of optical } \\
\text { performance. Change between fabrication and operation temperatures } \\
\text { must be considered }\end{array}$ \\
\hline
\end{tabular}

Of these, \#6 is typically the greatest challenge to the performance of the telescope. Since most optical surfaces are to be maintained to within nanometers of the prescribed form [2](with currently envisioned missions of HabEx, LUVOIR and LISA requiring surfaces constant to a few picometers), we are concerned with effects often ignored in less demanding engineering. Each material will be subject to both the innate material property, and also the process available to implement the mirror.

\section{A Materials checklist:}

Temperature: The surface difference between fabrication at room temperature and operation for systems going to cryogenic can be large. Special methods are necessary to accommodate such changes. However, many spaceborne telescopes operate near room temperature.

- Temperature gradients, and especially temperature transients are most demanding. Gradients are inevitably large while looking at astrophysical objects in cold space, and transients occur as the thermal view factors of the sun and earth on the telescope change. For earth observing at low earth orbits, while the earth is warm compared to cold space, thermal source view factors will modulate with orbital phase. The ability of the mirror material to accommodate gradients is crucial.

There are two approaches to managing thermal transients innate to material selection. Any deficiency of the thermal solution to address surface stability through material properties must be augmented by sensing temperatures and proportionally adding heat. Innate material stability depends on the thermal expansion over the thermal range, and the thermal diffusivity. Unfortunately, practical materials for mirrors have either a High Coefficient of Thermal Expansion (CTE) with a high Thermal Diffusivity (TD), or a low CTE with a low TD. Metals and ceramics fall in the first category, and low expansion glass and glass ceramics fall in the second category. Later in this paper, the relative effectiveness of each approach will be illustrated.

Best thermomechanical properties: Elastic modulus, density, homogeneity, isotropy, and dimensional instabilities resulting from changing thermal boundary conditions or sources and sinks all must be evaluated and the connections between these parameters must be analyzed.

- High Breakage resistance: Material strength, knowledge of that strength from large numbers of tests, and consistency of strength throughout the mirror volume should be among evaluation factors. Available data is key to making the proper decisions, as is the ability to inspect for subsurface defects. What is the dispersion of test results? 
- Maintain mirror alignment: In a telescope, one mirror is aligned to at least one more mirror, then to the focal plane. Optical tolerancing must consider the stability of this alignment metering through different operation temperatures than those in the laboratory where the system was assembled, integrated and tested. The availability of material of high strength that reasonably matches those characteristics of the mirror material is a selection factor, relating to both the telescope error budget, and the level of effort needed for supplemental thermal management.

\section{RFERENCE TO THE THERMAL ATTRIBUTES OF ZERODUR ${ }^{\circledR}$}

\section{Thermal expansion at different operating temperatures}

Thermal stability is a key requirement for most spaceborne systems. Because attributes of ZERODUR ${ }^{\circledR}$ have been extensively published by SCHOTT and others, we start by referencing our discussions to this available data. ZERODUR ${ }^{\circledR}$, a very low thermal expansion glass ceramic, illustrates critical attributes of materials in this class for thermal design. Figure 1 illustrates that modest thermal strain may be achieved even going between room temperature and $\mathrm{LN}_{2}$ temperatures. Furthermore, as we will describe later, the tailored expansion of ZERODUR ${ }^{\circledR}$ closely matches expansions that can be realized for the common structural material Carbon Fiber Reenforced Polymer.

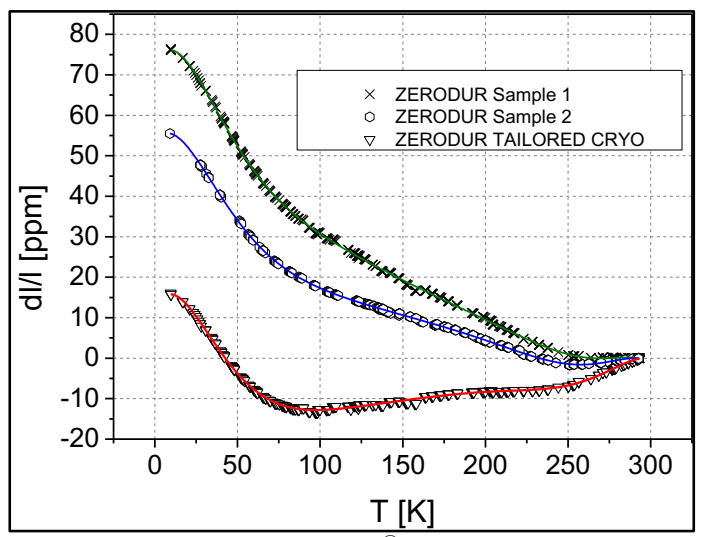

Figure 1. Without changing the heritage composition of ZERODUR ${ }^{\circledR}$ glass ceramic, the thermal expansion characteristics can be altered by process parameters in ceramization. In this example, $\mathrm{dl} / \mathrm{l}$ at Room Temperature is identical to $\mathrm{dl} / \mathrm{l}$ at $\sim 40 \mathrm{~K}$. The $\mathrm{dl} / \mathrm{l} \mathrm{curve}$ can be further optimized depending on the operational temperature or operational temperature range [3].

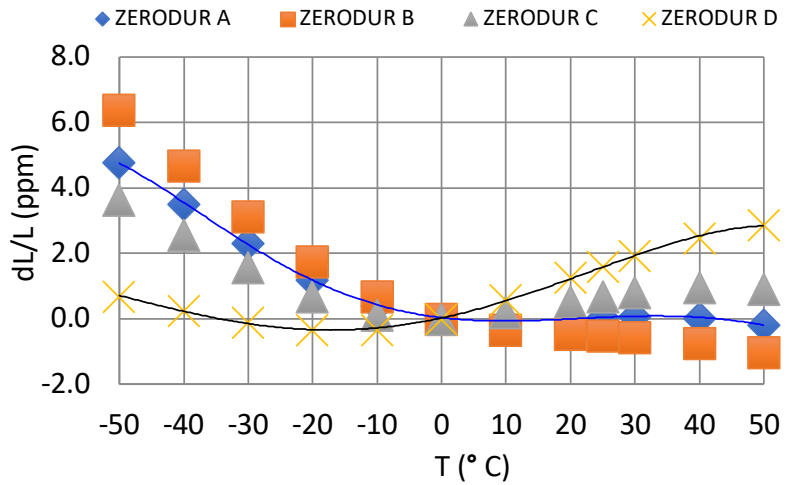

Figure 2. Here we illustrate 4 different measured target thermal expansion curves for ZERODUR ${ }^{\circledR}$. Note designations A,B,C,D are for convenience here and not SCHOTT defined profiles. Thus, if the telescope was to operate between $\mathrm{T}=0{ }^{\circ} \mathrm{C}$ and $\mathrm{T}=-30$ 
${ }^{\circ} \mathrm{C}$, ZERODUR D would be preferred. While if the telescope was to operate between $10{ }^{\circ} \mathrm{C}$ and $40{ }^{\circ} \mathrm{C}$, ZERODUR A would be preferred.

Many telescope assemblies operate over a shorter temperature range than that expressed in Figure 1. The ability to tailor the thermal expansion characteristics of ZERODUR ${ }^{\circledR}$ to various operational environment conditions simplifies the design requirements imposed on an Optical Telescope Assembly (OTA). The CTE of ZERODUR ${ }^{\circledR}$ may be optimally tailored over the shorter thermal range than that expressed in Figure 1, a thermal range often encounter in OTAs operating in the Visible and Ultraviolet (wavelengths between $100 \mathrm{~nm}$ and $1100 \mathrm{~nm}$ ). The tailoring is done in the production process and does not alter the composition or Technology Readiness Level (TRL) of the material. Thus, the material itself may largely provide required dimensional stability over orbital varying thermal boundary conditions. While this tailoring of ZERODUR ${ }^{\circledR}$ may be applied to optimally match operating conditions, in Figures 2 and 3 we express four examples of strain as a function of temperature for different factory applied tailoring, suggesting that a variety of thermal environments may be met with a high degree of passive stability.

Because there are maxima and minima in the strain curves of Figure 2, the derivative with temperature, coefficient of thermal expansion (CTE), will be zero at these temperatures. Ultraprecision mirrors may be designed to maintain the mirror close to one of these ZERO Crossing Temperature. To do this, a gradual slope of CTE with temperature is important. The larger the slope, the more homogeneous the material must be in CTE.

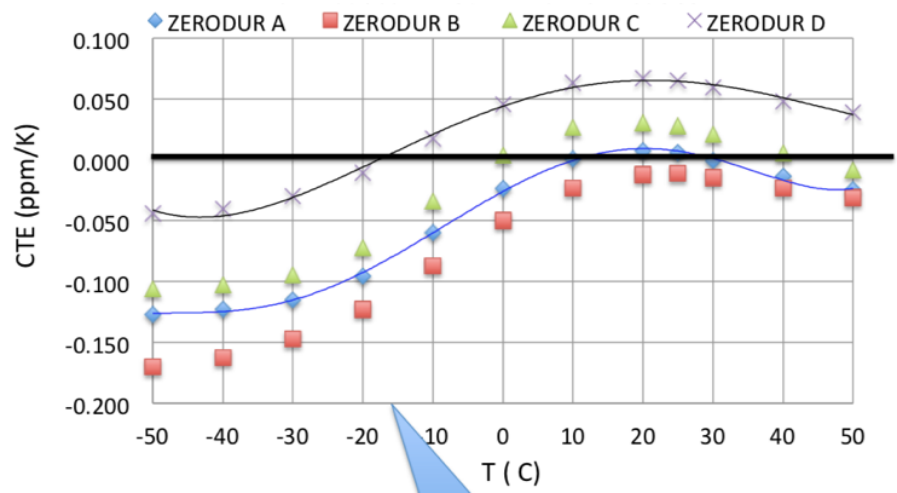

Figure 3. Designations A,B,C,D are converted into CTE. Note that the change of slope in Figure 2 implies that CTE can be both positive and negative, and that there are ZERO Crossings of CTE, as designated at $-17^{\circ} \mathrm{C}$ for case D.

ZERODUR $^{\circledR}$ is a monolithically cast material, distinct from a mirror substrate fabrication using multiple pieces, and from substrates built additively. As such, it is innately homogeneous as illustrated in the Figure 4 [A]. For a low expansion material like ZERODUR ${ }^{\circledR}$, characterization depends upon precision dilatometry at the part-per-billion/K (ppb/K) level [4]. CTE metrology accuracy to this level has been established at the ZERODUR ${ }^{\circledR}$ factory floor. This qualifies the substrate material for high precision telescopes. The achieved homogeneity is crucial for minimizing surface deviations associated with taking the mirror from room temperature to a different operational temperature, and also for consistent performance over the operation range. Furthermore, the product of (inhomogeneity* $\frac{\partial C T E}{\partial T}$ ) at the CTE ZERO crossing temperatures 
must be minimal if the mirror surface accuracy is to be actively maintained. The following figures illustrate measured
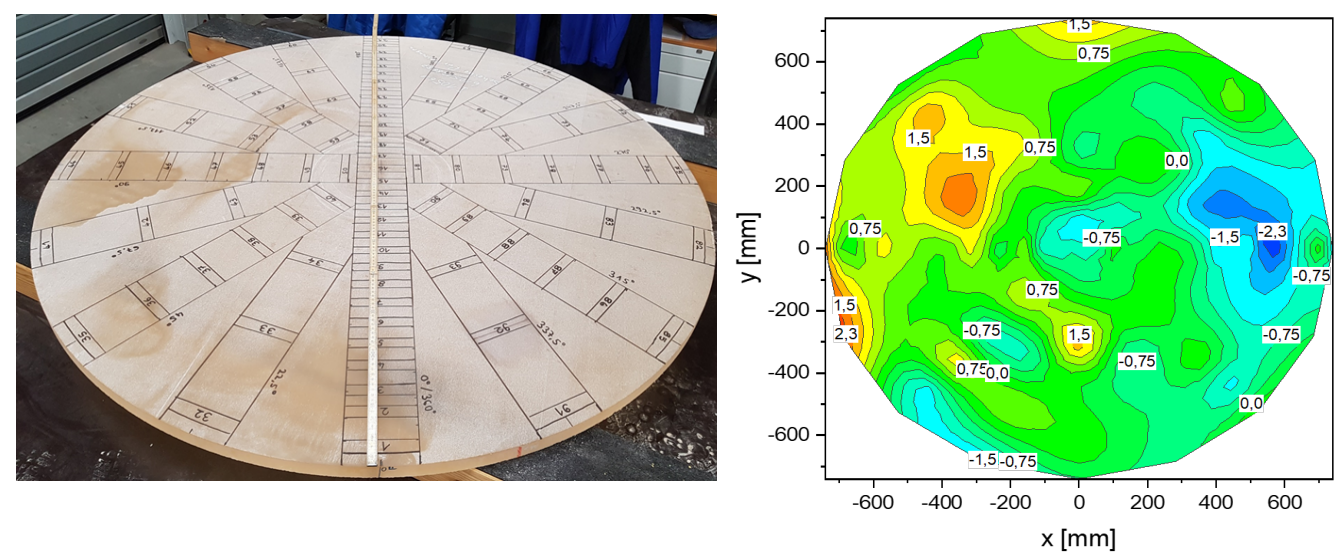

DCTE $[\mathrm{ppb} / \mathrm{K}]$

homogeneity of mirror blanks at various spatial frequencies.

Figure 4. The $1.5 \mathrm{~m}$ disk, $2 \mathrm{~cm}$ thick, is sampled in a polar coordinate sys tem exhibiting homogeneity of $5.3 \mathrm{ppb} / \mathrm{K}$ peak-to-valley, consistent with the mixing process associated with casting the blank

blank 1, measurement from 2006

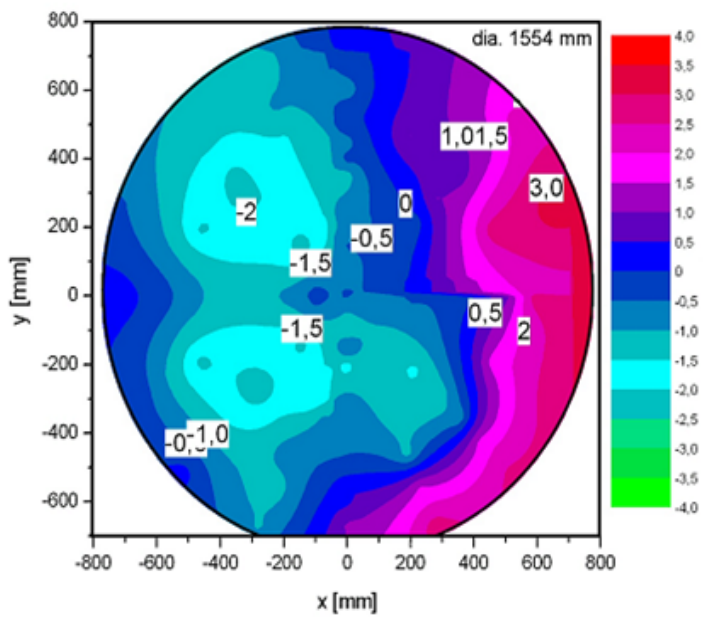

blank 2, measurement from 2008

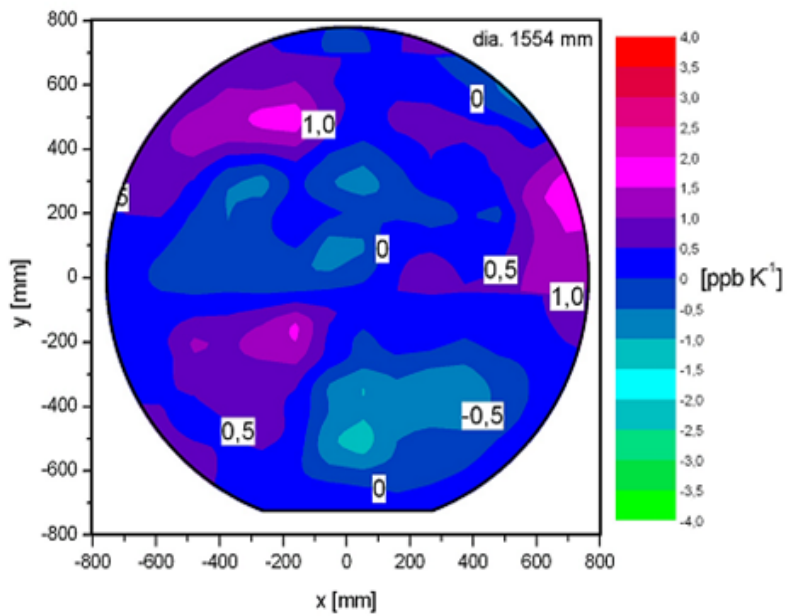

Figure 5: Homogeneity measurements made on two ZERODUR ${ }^{\circledR} 1.554 \mathrm{~m}$ diameter blanks produced three years apart further establishes the low level of inhomogeneities in the process [5]. The measures in Figure 4 and Figure 5 establish homogeneity on moderately large blanks. These are sampled at of the order of $20 \mathrm{~cm}$ average increment. Recently, measurements have been made of material around a full $4 \mathrm{~m}$ blank, exhibiting very small inhomogeneity. Smaller scales are highly relevant to critical stability. Sacrificial measurements have also been made of a $10 \mathrm{~cm}$ cube of ZERODUR ${ }^{\circledR}$ sampling on the $1 \mathrm{~cm}$ spatial scale. Results are found in Figure 6 . In summary, Table 2 summarizes homogeneity on various size blanks and sampling at various spatial scales. 


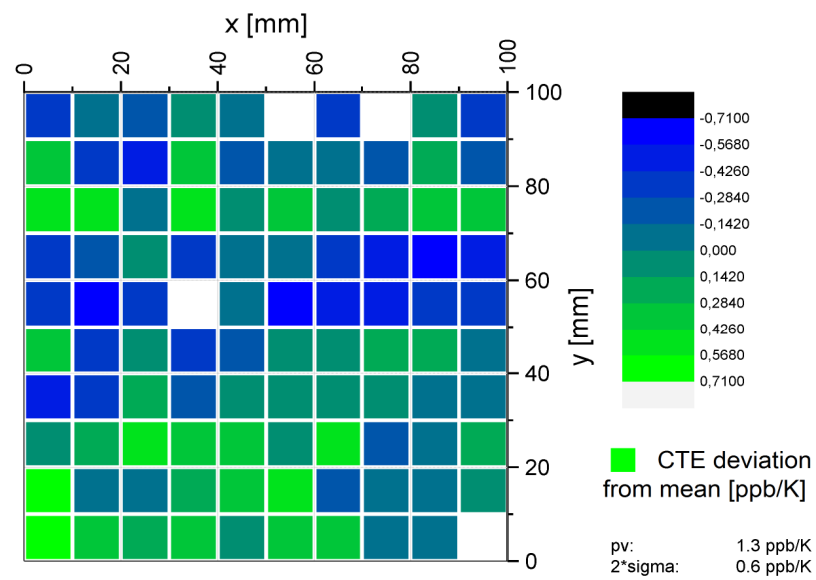

Figure 6. Homogeneity is established at the $1 \mathrm{~cm}$ scale for ZERODUR ${ }^{\circledR} .97$ coupons fell within a peak-to-valley range of $1.3 \mathrm{ppb} / \mathrm{K}$.

Table 2. Measured CTE Homogeneity [5]

\begin{tabular}{|l|l|l|l|}
\hline Piece & Size & Spatial Scale & P-to-V CTE \\
\hline Cube & $10 \mathrm{~cm}^{*} 10 \mathrm{~cm}^{*} 10 \mathrm{~cm}$ & $\sim 1 \mathrm{~cm}$ & $1.3 \mathrm{ppb} / \mathrm{K}$ \\
\hline Disk & $154.4 \mathrm{~cm} \mathrm{dia} * 2 \mathrm{~cm}$ & $\sim 20 \mathrm{~cm}$ & $5.3 \mathrm{ppb} / \mathrm{K}$ \\
\hline 2006 Blank & $1.5 \mathrm{~m} \mathrm{dia}$ & $\sim 20 \mathrm{~cm}$ & $6.4 \mathrm{ppb} / \mathrm{K}$ \\
\hline 2008 Blank & $1.5 \mathrm{~m} \mathrm{dia}$ & $\sim 20 \mathrm{~cm}$ & $3.8 \mathrm{ppb} / \mathrm{K}$ \\
\hline Blank & $4.0 \mathrm{~m} \mathrm{dia}$ & $\sim 20 \mathrm{~cm}$ & $4.0 \mathrm{ppb} / \mathrm{K}$ \\
\hline
\end{tabular}

NASA MSFC conducted optical tests of a SCHOTT provided lightweighted mirror at the XRCF thermal vacuum facility. The mirror substrate was 1.2 in diameter, lightweighted $88 \%$ and prepared as a machining demonstration. The mirror was polished as a sphere, and kinematic mounts added. MSFC conducted interferometric tests at temperatures $295 \mathrm{~K}, 270 \mathrm{~K}$, $250 \mathrm{~K}$ and $230 \mathrm{~K}$ [6]. The homogeneity maps were not available, NASA's analysis attributed all the small changes in mirror shape to inhomogeneity, even though gradients and other systematic errors were known to be present. Even with this assumption, all models of homogeneity suggested that the inhomogeneity could be no larger than 5ppb, in agreement with SCHOTT dilatometry maps on $1.5 \mathrm{~m}$ disks.

\section{Relative thermal merit of various mirror materials}

For optimal telescope optical performance, two conditions are to be met as the telescope or instrument assembly goes through gravity release, launch loads, and operating temperatures over a range often not including the fabrication temperature.

1. Mirror surfaces should represent within error budget tolerance their as-polished shape through all relevant environments and over time. This includes changes in radius of curvature as well as irregularity through the first 36 Zernike polynomials, and Mid-Spatial Frequencies (MSF) usually considered between 3 and 30 or more cycles per aperture.

2. Metering structure must maintain within error budget tolerance the prescribed relative position of each mirror and focal plane, again over all relevant environments and over time. 
In most telescopes of moderate to large size range, there is a provision for focusing, and perhaps adjustments of other degrees of freedom once on orbit. Exoplanet coronagraphs and other critical narrow angle systems may even reimage the entrance pupil to restore low- and mid-spatial frequency errors to ideal form.

Our consideration is passive athermalization, the extent that the mirror material may minimize errors without mechanical intervention or proportional addition of heat at nodes. A common comparison is often evoked comparing CTE to thermal diffusivity ( $=$ thermal conductivity/(density $\left.{ }^{*} c_{\mathrm{p}}\right)$ ). Thermal diffusivity is a measure of the volume of material to come to a new equilibrium temperature. We consider three cases:

A. Materials exhibiting High Thermal Diffusivity accompanied with High CTE. Examples of such materials are Aluminum, Beryllium and Silicon Carbide. As temperature changes, the radius of curvature of the mirrors will also change accommodating the change in spacing between the mirrors.

B. Materials exhibiting Low CTE accompanies with LowThermal Diffusivity. Examples are Corning ULE, Ohara CLEARCERAM and SCHOTT ZERODUR ${ }^{\circledR}$.

C. Materials exhibiting high Thermal Diffusivity with a steep CTE slope through a temperature where CTE is zero. This may be used only when homogeneity is very high, and the system will employ high authority active proportional heating

The thermal stability ideal might evoke mirror materials exhibiting both High Thermal Diffusivity and Low CTE over typical broad operational temperature ranges. Unfortunately, such materials are not available. Therefore, a trade is needed among available materials based on consideration of

- Thermal Diffusivity

- $\quad$ CTE at operational temperatures

- For active thermal control

Rate of change of CTE with temperature

- Homogeneity of CTE

Materials exhibiting High Thermal Diffusivity with High CTE can be made to work only if isothermal conditions are maintained. However, the sun and earth expose a heat load on part of the telescope, while cold space constitutes a thermal sink. Typically, this is a transient situation as well, depending on whether the observatory is earth observing or conducting astrophysics. For such a system, can the system become sufficiently isothermal rapidly enough or at all from spaceborne boundary conditions, even if heat is added? This is especially true for diffraction limited telescopes that operate in the visible and ultraviolet part of the spectrum. It is less true for telescopes at long wave infrared and sub-millimeter wavelengths that have much more forgiving optical tolerances.

Our focus is on Cases A and B above, which address most mirror material solutions currently being considered. Case $\mathrm{C}$ is an emerging solution being posed by Cordierites for cases that include high authority temperature control of the mirror, addressing the challenge of steep slope of CTE(T) at the temperature of CTE zero crossing, authority of control and CTE slope driven demands on homogeneity. The numerical simulation in Figure 7 examines the critical trade between Cases A and $\mathrm{B}$ above [7,8]. Intentionally, a thermally stressing orbit was modelled with identical telescopes, one with the primary mirror of $\mathrm{SiC}$ and the other with the primary mirror of ZERODUR ${ }^{\circledR}$. The analysis assumed an 100-minute low earth orbit, with the telescope nadir looking toward Earth. Furthermore, the orbit is made to pass through the earth's umbra, inducing thermal perturbation in addition to a solar view factor rotating $360^{\circ}$ per orbit. In this case, the Low Thermal Diffusivity, Low CTE selection performs substantially better than the $\mathrm{SiC}$ case. 


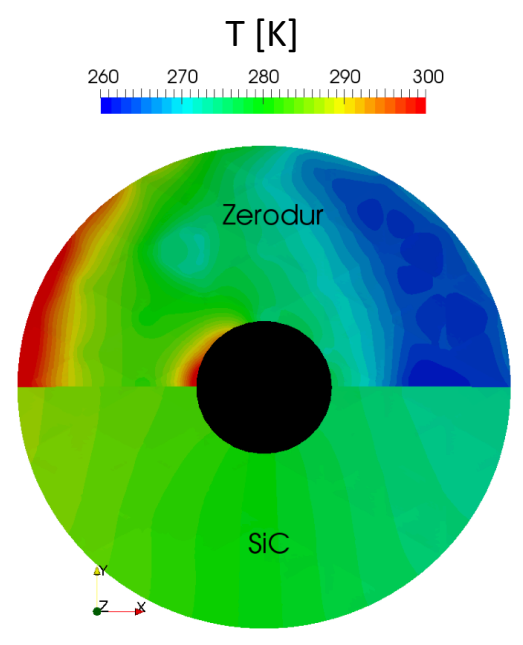

t: $2200 \mathrm{~min}$
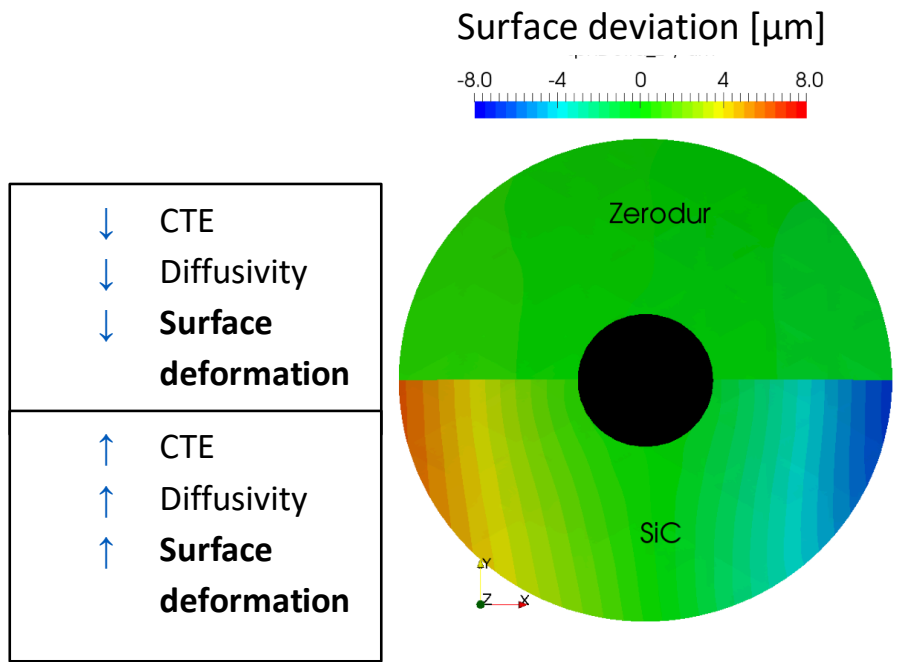

Figure 7. This simulates the relative response of a ZERODUR ${ }^{\circledR}$ and a SiC primary mirror in telescopes, identical except for the material. After 22 orbits, the temperature distribution on the primary mirror of each telescope is examined (Left), and as expected, temperature is notably smoother for $\mathrm{SiC}$. However, the opposite is true for surface deviation (Right). This demonstrates that in comparison with an extremely low expansion material like ZERODUR ${ }^{\circledR}$, high diffusivity fails to compensate for ZERODUR ${ }^{\circledR}$ 's low CTE in terms of surface deviation. [7,8]

A number of mirror materials have been used in spaceborne telescopes, each with strengths and weaknesses. The attributes a designer considers are broad. First, the material must have the dimensional stability in the environment satisfying the imaging performance requirements. Principal among these is dimensional stability in the orbital environment. Heritage, Technology Readiness Level (TRL), and mechanical strength and mass attributes are all important, as is the ability of the material to take a smooth optical surface. Increasingly, telescope cost and manufacture schedule are becoming deciding factors, especially for moderate sized telescopes in the $0.3-1 \mathrm{~m}$ diameter class. 
Table 3. Material Properties Comparison I: Glass ceramics, glass, cordierites and metals. Bold designates favored.

\begin{tabular}{|c|c|c|c|c|c|c|}
\hline \multirow{2}{*}{$\begin{array}{l}\text { Attribute / } \\
\text { Material }\end{array}$} & \multirow{2}{*}{$\begin{array}{l}\text { Maximum } \\
\text { monolithic } \\
\text { diameter }\end{array}$} & \multicolumn{4}{|c|}{ CTE } & \multirow{2}{*}{$\begin{array}{l}\text { Inspect } \\
\text { volume }\end{array}$} \\
\hline & & Value & $\begin{array}{l}\text { Homo- } \\
\text { geneity }\end{array}$ & $\begin{array}{c}\text { 0 CTE } \\
\text { crossing }\end{array}$ & $\begin{array}{l}\text { CTE Slope @ } \\
\text { o Crossing }\end{array}$ & \\
\hline ZERODUR ${ }^{\circ}$ & $4.3 \mathrm{~m}$ & $\begin{array}{c}\text { Extremely Low } \\
5 \mathrm{ppb} / \mathrm{K}\end{array}$ & Superb & $\begin{array}{l}\text { Yes, most } \\
\text { cases }\end{array}$ & Low & $\begin{array}{l}\text { Visible } \\
\text { high } \\
\text { resolution }\end{array}$ \\
\hline ULE & $\begin{array}{c}\sim 1.5 \mathrm{~m} / \text { fuse } \\
\text { larger }\end{array}$ & Low $30 \mathrm{ppb} / \mathrm{K}$ & $\begin{array}{c}\text { Not } \\
\text { published }\end{array}$ & Yes & Low & $\begin{array}{l}\text { Visible } \\
\text { high } \\
\text { resolution }\end{array}$ \\
\hline Fused Silica & $\sim 1.5 \mathrm{~m}$ & $550 \mathrm{ppb} / \mathrm{K}$ & Good & No & NA & $\begin{array}{l}\text { Visible } \\
\text { high } \\
\text { resolution }\end{array}$ \\
\hline Cordierite 720 & $\sim 1.5 \mathrm{~m}$ & $\begin{array}{l}\text { Moderate } \\
\text { Variable }\end{array}$ & $\begin{array}{c}\text { Not } \\
\text { published }\end{array}$ & Yes & High & $\begin{array}{l}\text { X-ray low } \\
\text { resolution }\end{array}$ \\
\hline $\mathrm{SiC}$ & $\begin{array}{c}\sim 1.5 \mathrm{~m} / \text { fuse } \\
\text { larger }\end{array}$ & $2500 \mathrm{ppb} / \mathrm{K}$ & Variable & No & NA & $\begin{array}{l}\text { X-ray low } \\
\text { resolution }\end{array}$ \\
\hline Al & $\sim 0.4 \mathrm{~m}$ & $23000 \mathrm{ppb} / \mathrm{K}$ & Variable & No & NA & $\begin{array}{l}\text { X-ray low } \\
\text { resolution }\end{array}$ \\
\hline $\mathrm{Be}$ & $\sim 1.6 \mathrm{~m}$ & $12000 \mathrm{ppb} / \mathrm{K}$ & Variable & No & NA & $\begin{array}{l}\text { X-ray low } \\
\text { resolution }\end{array}$ \\
\hline
\end{tabular}

Table 3 emphasizes the parameter maximum monolithic diameter. Also, a consideration is the ability to inspect for subsurface defects. If voids or inclusions manifest during machining a lightweight mirror, or optically finishing the mirror, the expense and lapse time of replacing the material in these operations may be considerable. For many materials, mirrors are made of joined sections of different material production runs. For example, the $3.5 \mathrm{~m}$ Herschel Telescope primary mirror was made of 9 pieces of $\mathrm{SiC}$, each brazed together. This piecing together of pieces of material made in differing batches introduces a number of uncertainties in response to thermal stimuli. In the case of Herschel, operation was between the wavelengths of 80 microns to 640 microns. Since the wavelength is approximately two orders of magnitude larger than telescopes operating at visible wavelengths, the error is proportionally more forgiving to effects of joining materials, and of any dissimilarities between the 9 parts in thermal response. In a different manner, large ULE mirror blanks may frit bond or low temperature fuse a number of pieces of ULE to make a single mirror (for example the $1.8 \mathrm{~m}$ Technology Demonstration Mirror made for NASA joined by low temperature fusion 9 separate pieces of ULE to form the $1.8 \mathrm{~m}$ diameter mirror [9]. This mirror was never completed, and the effects of joining pieces is not known.). In the case of aluminum, while castings can be made quite large, preferred materials for diamond turned mirrors require rapid quenching, as done by RSP Technology, and it appears that this quenching cannot be homogeneous for large mirrors. .

For materials that take an optical surface or can be clad with another material to take a smooth optical surface, considerations for spaceborne mirrors center on mass efficiency, and resilience to thermal transients. These are often plotted against each other (Figure 8). Mass characteristics have been important in situations when the space element mass is required to be limited to meet the capacity of launch vehicles reaching the desired orbit. A useful measure of this is the quotient of $\mathrm{E}$, the modulus of elasticity divided by the density, $\rho$. High modulus materials can make thinner mirrors with the same degree of deflection in gravity. Beryllium has both a very high $\mathrm{E}$ and a low $\rho$, favorable from the structural point of view. This ratio is less critical presently with the emergence larger lift capacity launch vehicles. While E/ $\rho$ represents bending, once in space the eigenfrequency of vibration response to stimulus from cryocoolers or spacecraft reaction wheels may be more important. The fundamental eigenfrequency $f_{0}$ scales with $\sqrt{ }(E / \rho)$. 
The resilience to thermal transients is expressed as Thermal Diffusivity $D\left(D=\right.$ Thermal Conductivity/(density $\rho^{*}$ specific heat at constant pressure $\mathrm{c}_{\mathrm{p}}$ ) divided by the CTE (or $\alpha$ ).

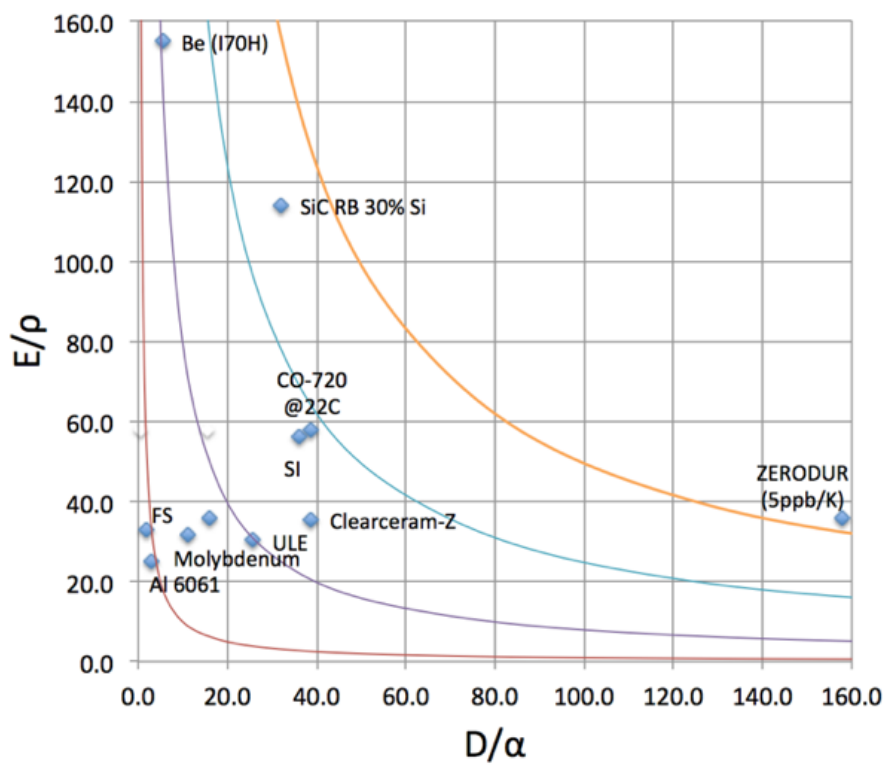

Figure 8. Frequently, the first order figures-of-merit for candidate mirror materials are taken to be the material's structural efficiency (Elastic Modulus divided by Density, the metric for gravitational or inertial displacement) and its Thermal Transient Resilience (Thermal Diffusivity D divided by CTE ( $=\alpha$ in the chart) ( where $\mathrm{D}=$ thermal conductivity divided by the product density times specific heat $\mathrm{cp}$ ). Here, we show one plotted against the other. The hyperbolic contours are for $(E / \rho)^{*}(D / \alpha)=$ constant implying these two metrics are of equal weight. Frequently today, the Abscissa giving the thermal figure of merit is favored. Here FS=Fused Silica, CO-720=Cordierite, and $\mathrm{Si}=$ Silicon. ZERODUR ${ }^{\circledR}$, with both $\mathrm{CTE}$ and CTE homogeneity as low as $5 \mathrm{ppb} / \mathrm{K}$, is in a favored position for thermal response. Since eigenfrequency relating to vibration response goes as $\sqrt{ }(E / \rho)$, the separation along the ordinate is less.

\section{MIRROR SUBSTRATES FOR STRENGTH AND LIFETIME}

Peter Hartmann [10,11] has conducted and published extensive experiments on the breakage stress and lifetime of ZERODUR ${ }^{\circledR}$. These are summarized in Figure 9.
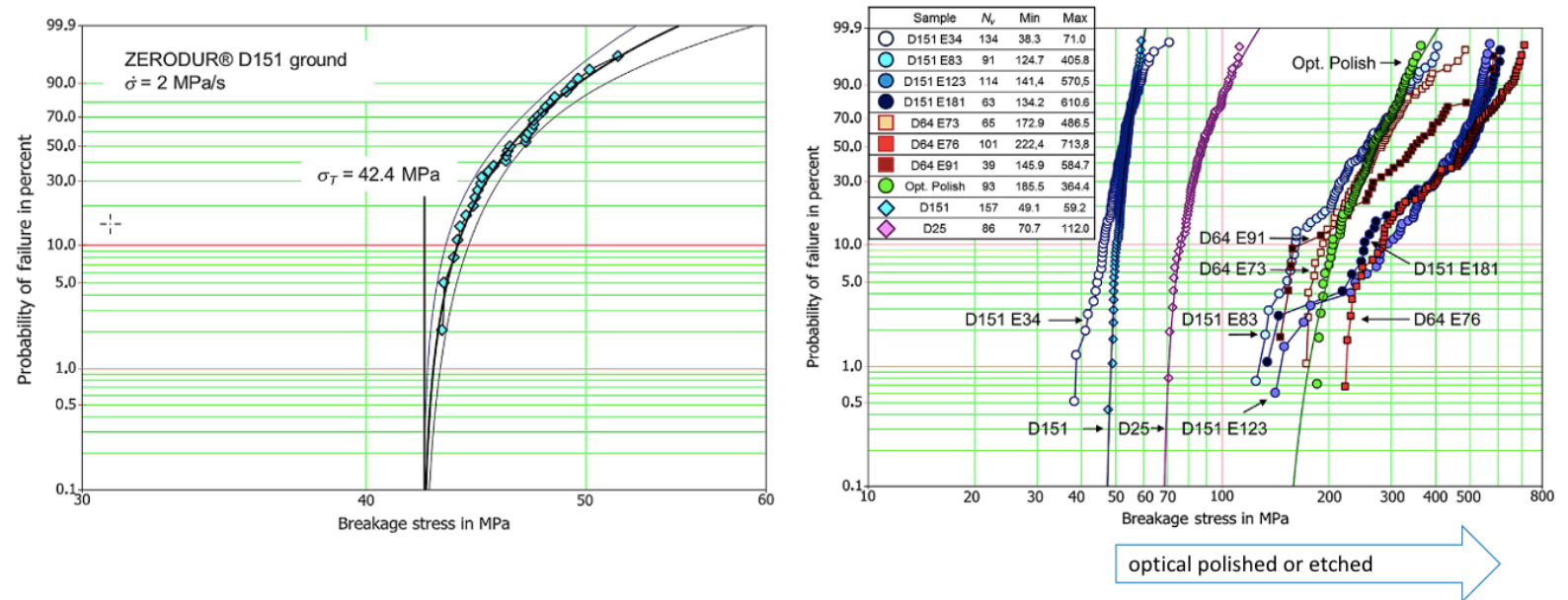

Figure 9: Determination of threshold stress from breakage data for different surface conditions by fitting the 3-parameter Weibull statistics 
This constitutes statistically sound primary source of engineering data for design with ZERODUR ${ }^{\circledR}$. Note that the result of breaking a large number of samples demonstrates the applicability of three parameters to Weibull statistics that govern the threshold stress exposure a properly prepared part of ZERODUR ${ }^{\circledR}$ may experience without breakage. The threshold Breakage Stress of $42.4 \mathrm{MPa}$ is measured as the probability of failure goes asymptotically to zero. The threshold Breakage Stress is increased as finer grits are used in fixed abrasive machining, as the samples are prepared with acid etched surfaces to remove sub-surface damage from machining, and as surface damage is removed optically by progressive grit loose abrasive grinding.

From this study, lifetime predictions have been conducted and published by Hartmann [11].

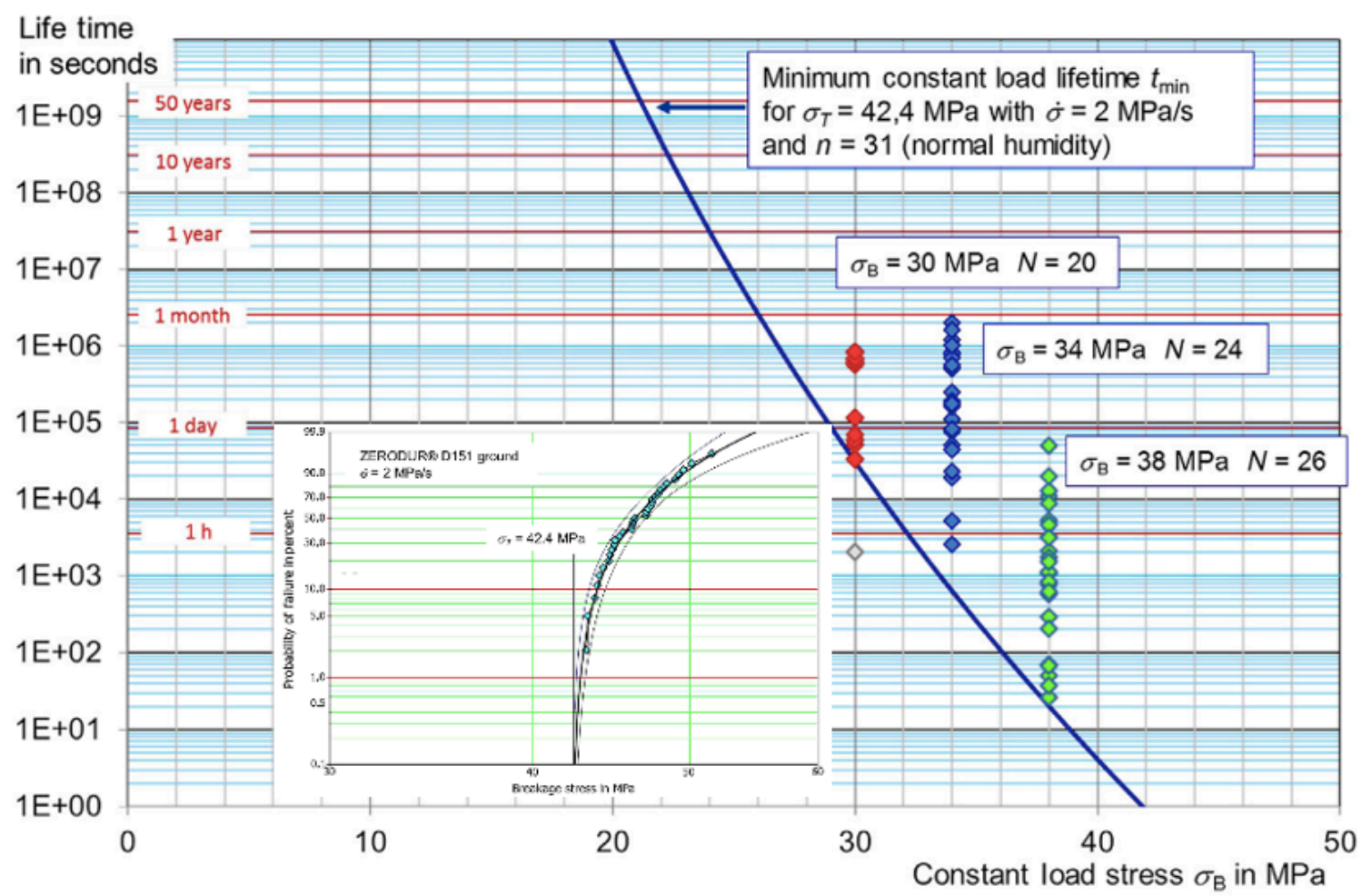

Figure 10: For ZERODUR ${ }^{\circledR}$, the designer can confirm the component's lifetime based on 3-parameter Weibull Statistic and the stress corrosion coefficient. The threshold values of bending strengths are derived, a lifetime calculation is made including the theory of crack corrosion. Then the lifetimes curves are confirmed with three experimental setups, each with constant load. No breakages were observed to the left of the lifetime curve, confirming the theory.

The values sited in Figures 9 and 10 are from a cast material. Different materials should be expected to exhibit quite different breakage statistics, and different dispersions in breakage test values. We have compared the distribution of breakage stress in ZERODUR ${ }^{\circledR}$, a cast material with the distribution of breakage stress in a sintered material. Figure 11 illustrates the larger dispersion of breakage stress between the materials. This means that a significantly larger margin of safety may be necessary to be applied to a sintered material compared to that to a cast material. 


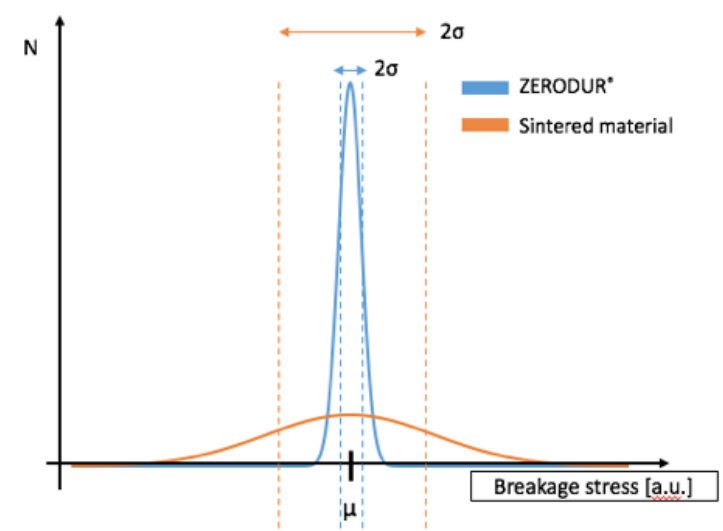

Figure 11: Comparing the breakage stress dispersion of a sintered material to that of ZERODUR ${ }^{\circledR}$, a cast material.

\section{THE TELESCOPE STRUCTURE RELATES TO MIRROR MATERIAL}

The success of the optical system depends on both the mirror stability, and also that of the metering structure. A high expansion material like aluminum, beryllium and $\mathrm{SiC}$ exhibit strength sufficient to make the metering structure out of the same material as the mirror, a mono-material approach. In some cases, LISA Pathfinder for example, precision optical benches and even telescope structures are made of low expansion materials like ZERODUR ${ }^{\circledR}$, thus having the monomaterial approach. High-strength structural materials may be used to match in many ways the thermal expansion characteristics of the mirror material. In the case of low expansion materials, Carbon Fiber Reenforced Polymers (CFRP) are frequently used. CFRP is an orthotropic planar material, with the CTE of the two axes in the plane similar, and the third axis, not maintaining the metering, may be quite different. For a CFRP like M55J, the thermal expansion characteristics in the plane can be tailored to match the substrate [3]. As described in Section 2, ZERODUR ${ }^{\circledR}$ is routinely tuned to the mission's expected environment, and also to the metering material. Figure 12 illustrates ZERODUR ${ }^{\circledR}$ being tailored to match in thermal strain to a nominal M55J between room temperature and $\mathrm{LN}_{2}$ temperatures.

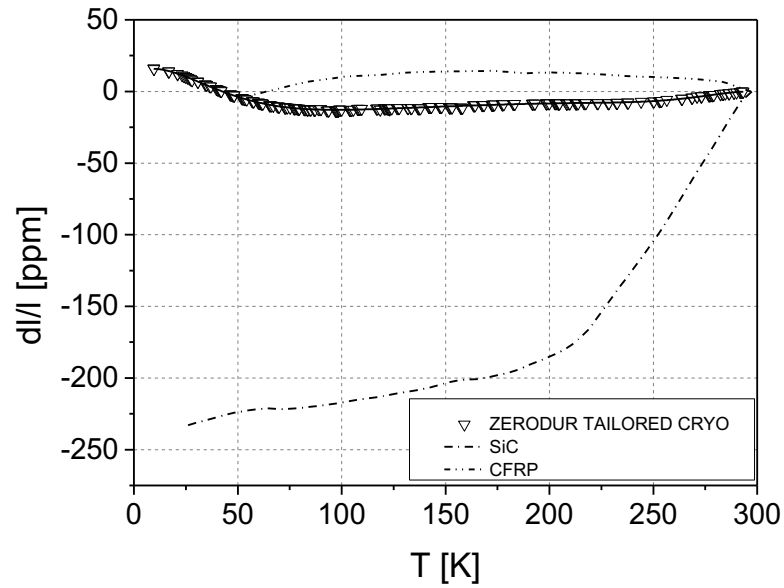

Figure 12: ZERODUR ${ }^{\circledR}$ tailored to match the strain of CFRP between room temperature and $\mathrm{LN}_{2}$ temperatures

While this demonstrates the viability of mounting a low expansion mirror material on a low expansion metering material, there are further possibilities. In this example, the match was over a long temperature range down to cryogenic 
temperatures. However, many applications maintain the mirror within $20^{\circ} \mathrm{C}$ to $30^{\circ} \mathrm{C}$ below room temperature. Over a shorter range, since both materials are tailorable, the strain curve for ZERODUR ${ }^{\circledR}$ may be co-adjusted to the strain curve for CFRP, leaving very little differential metering strain. This is a step toward minimizing the cost, risk and complexity associated with high authority thermal control.

\section{SUMMARY: PERFORMANCE OF SPACEBORNE MIRROR MATERIALS}

The implementation of a successful spaceborne telescope must address controlling dimensional instabilities into ordersof-magnitude smaller regimes than traditional engineering applications. Every design decision is critical, and the choice of mirror material is especially critical. Material selection is often one of the earliest decisions in the design process, and the effects resulting from this choice propagates throughout the production and operation of the assembly. This choice of mirror material invariably impacts the performance, complexity and cost figures-of-merit of the optical system.

The best design must look beyond the first order characteristics of a material like the elastic modulus, density, thermal conductivity and CTE. Other aspects are crucial. To name a few:

- Is the substrate homogeneous in CTE to a few parts-per-billion/K over the entire volume?

- Is the material anisotropic?

- Does microyield or microcreep take place?

- Are there Weibull statistics to give a true measure of design loads, lifetime and breakage resistance?

- Are radiation characteristics appropriate error budget allocations in the environment?

- Is the material transparent and offer opportunity for $100 \%$ inspection?

- Does the material exhibit the ability to be polished to a smooth surface without cladding?

- Can the material CTE be tailored to minimum in the operational range?

- Can the material work in a system where thermal gradients are present?

- Does the material offer space heritage for the wavelength and application, and associated high Technology Readiness Level?

\section{ACKNOWLEDGEMENTS}

We wish to thank many technical people at SCHOTT for computational support and technical discussions. These include Dr. C. Kunisch, A Leys and Dr. P. Hartmann. We also thank Dr. Phil Stahl of NASA for his interest in ZERODUR ${ }^{\circledR}$, and his support in undertaking substantial NASA tests of lightweighted ZERODUR ${ }^{\circledR}$ mirror blanks.

\section{REFERENCES}

[1] Tribble, A. [The Space Environment], Princeton University Press, Princeton, NJ (1999)

[2] Stahl, H. Phillip et al, "Habitable-zone exoplanet observatory (HabEx) baseline 4-m telescope design and predicted performance", Proc. SPIE11115 (2019)

[3] Jedamzik, R., and T. Westerhoff, “ZERODUR TAILORED for Cryogenic Applications”, Proc. SPIE 9151 (2014)

[4] Jedamzik, R., Engel, A., Kunisch, C., Westenberger, G., Fischer, P., \& Westerhoff, T. (2015, September). Next generation dilatometer for highest accuracy thermal expansion measurement of ZERODUR ${ }^{\circledR}$. In Material Technologies and Applications to Optics, Structures, Components, and Sub-Systems II (Vol. 9574, p. 95740O). International Society for Optics and Photonics.

[5] Jedamzik, R. et al, “CTE Homogeneity of ZERODUR® in the ELT century”, Proc. SPIE11451-284 (2020)

[6] Brooks, T, Ron Eng, Tony Hull, Phil Stahl... "Modeling the Extremely Lightweight ZERODUR Mirror (ELZM) thermal soak test" Proc. SPIE 10374 (2017)

[7] T. Hull et al., presented at SPIE Security and Defense, Toulouse, 9648-10 (2015)

[8] T. Hull, T. Westerhoff, G. Weidmann, "Selection considerations between ZERODUR ${ }^{\circledR}$ and silicon carbide for dimensionally-stable spaceborne optical telescopes in two-earth-orbits" Proc. SPIE. Vol. 9573 (2015) 
[9] Cohen, E and T. Hull, "Selection of a mirror technology for the 1.8-m Terrestrial Planet Finder demonstrator mission", Proc. SPIE 5494 (2004)

[10]P. Hartmann, Proc. SPIE 10706-25 (2018 and references therein

[11] P. Hartmann, "Minimum lifetime of ZERODUR ${ }^{\circledR}$ structures based on the breakage stress threshold model: a review", Optical Engineering Vol. 58, Issue 2 (Feb 2019)

[12] Carré, A., Westerhoff, T., \& Hull, T. (2018, July). Impact of ionizing radiations on ZERODUR. In Space Telescopes and Instrumentation 2018: Optical, Infrared, and Millimeter Wave (Vol. 10698, p. 106981S). International Society for Optics and Photonics. 